\title{
Prevalence of Helicobacter pylori infection among dyspeptic patients in Northwestern Romania: A decreasing epidemiological trend in the last 30 years
}

\author{
ALEXANDRA LOOR COROJAN ${ }^{1}$, DAN-LUCIAN DUMITRAȘCU ${ }^{1}$, \\ PETRICĂ CIOBANCA $^{2}$ and DANIEL-CORNELIU LEUCUTA ${ }^{3}$ \\ ${ }^{1}$ Second Medical Department, 'Iuliu Hatieganu' University of Medicine and Pharmacy, 400006 Cluj-Napoca; \\ ${ }^{2}$ Central Laboratory, 'Salvosan Ciobanca' Medical Center, 450112 Zalau; ${ }^{3}$ Department of Informatics, \\ 'Iuliu Hatieganu' University of Medicine and Pharmacy, 400006 Cluj-Napoca, Romania
}

Received June 2, 2020; Accepted July 2, 2020

DOI: $10.3892 / \mathrm{etm} .2020 .9024$

\begin{abstract}
Infection with Helicobacter pylori (HP) has an unknown prevalence in several Romanian regions. Recent data are missing. The aim of this study was to estimate the prevalence of dyspepsia in the North-West part of Romania and to analyze the epidemiological trends of HP infection prevalence in a symptomatic population in this region by comparing with previous published data. Our study population consisted of 414 patients: 264 female $(63.8 \%)$ and 150 male (36.2\%), mean age $45.89 \pm 17.24$ years (range, 6-97 years) who attended a single secondary center in Zalau, Salaj, North-West Romania, between 2014 and 2018 for dyspeptic symptoms, either by their own initiative or by referral from their general practitioner. Testing was performed by IgG anti-HP assessment $\mathrm{G}$ anti-HP antibodies were found in 169 individuals (40.8\%). In females, the prevalence of HP infection was $40.53 \%$ (107/264) and in males $41.35 \%$ (62/150). There was a higher prevalence of positive antibodies in the rural areas compared with urban areas (42.29 vs. 39.75\%). In conclusion, the prevalence of HP infection is $40.8 \%$, without sex differences in dyspeptic patients from a representative population in North-Western Romania and the prevalence increases with age. Comparing our results with those of previous studies on the prevalence of HP infection from the same region, we were able to signal a decline in prevalence in HP infection over a 30 -year interval.
\end{abstract}

Correspondence to: Professor Dan-Lucian Dumitrascu, Second Medical Department, 'Iuliu Hatieganu' University of Medicine and Pharmacy, 2-4 Clinicilor Street, 400006 Cluj-Napoca, Romania E-mail: ddumitrascu@umfcluj.ro

\section{Abbreviations: HP, Helicobacter pylori}

Key words: Helicobacter pylori, dyspepsia, anti-Helicobacter pylori antibodies, prevalence, infection

\section{Introduction}

Helicobacter pylori (HP) infection is one of the world's most common infections. HP, a Gram-negative bacteria, is a human pathogen that is transmitted from human to human, and causes chronic active gastritis in all colonized subjects. This infection can lead to peptic ulcers, atrophic gastritis, gastric adenocarcinoma, and mucosa-associated lymphoid tissue (MALT) lymphoma. Therefore, HP is considered an infectious disease regardless of clinical severity (1).

Routes of transmission are considered: direct contact between subjects (2), contaminated water sources or food (3-5), zoonotic transmission and iatrogenic transmission during endoscopies and dental care (6).

$\mathrm{HP}$, is an intensely studied bacteria and plays a very important role in the ethiopatogenesis of gastric cancer, possibly carried by more than half of the world population (7).

The latest data regarding HP infection in Europe are from 2018 where it is shown that the lowest infection prevalence was found in Northern Europe, while the highest was in Eastern and Southern Europe, up to $84 \%$ in Portugal and Poland (8). Another systematic review and meta-analysis published in 2016 showed that Africa had the highest pooled prevalence of HP infection (70.1\%; 95\% CI, 62.6-77.7), whereas Oceania had the lowest prevalence (24.4\%; 95\% CI, 18.5-30.4). Among individual countries, the prevalence of HP infection varied from as low as $18.9 \%$ in Switzerland (95\% CI, 13.1-24.7) to $87.7 \%$ in Nigeria (95\% CI, 83.1-92.2) (9).

A review regarding the HP infection prevalence trends in Europe over time from 1990 to 2014 was published in 2015. The study reviewed the prevalence of HP across 35 European countries using surveys of unselected population. It showed that the prevalence of HP was much lower in northern and western regions of Europe than in eastern and southern Europe. The review revealed a significant reduction in the prevalence of HP over time with an overall mean reduction of $3.1 \%$ per year. Statistics showed that HP prevalence increased over younger age groups, often sharply, but levelled off in many studies from ages of $\sim 50$ years onwards, especially in areas of high prevalence. 
Regarding gastric cancer incidence in Europe, in the 15-year period from 1993 to 2007, there was also a moderate to large reductions over time in each of the 18 countries in which the studies were done (10).

The first report on prevalence of HP infection in Romania was published in 1990 (11). Helicobacter-like organisms were identified using histological staining from antral gastric mucosa: $72.8 \%$ in gastric ulcer, $69.6 \%$ in duodenal ulcer, $69.2 \%$ in bulbitis, $61 \%$ in chronic gastritis, $50 \%$ in gastric cancer and $34.3 \%$ in healthy controls.

Currently there are various methods used for HP detection in all categories of patients, but it is recommended to use only those methods that have high specificity and sensitivity. The IgG anti-HP antibodies have low sensitivity because they can persist in blood plasma in high levels for many years even after treatment. Studies show that levels of IgG anti-Hp antibodies in the serum do not predict the presence of macroscopic gastroduodenal diseases or the density of HP colonization in HP-infected dyspeptic patients. In addition, there are some levels which do not allow a precise determination of HP status (12-15). Thus, the presence of anti-HP antibodies in the serum indicates that the patient had contact with the bacteria, without being able to determine definately whether the infection still exists.

The specific objectives of this study were: i) to estimate the prevalence of HP exposure in dyspeptic patients who presented to the hospital from this northwestern region of the country; ii) to determine the association of the HP exposure with potential risk factors such as age, sex and the area of residence; iii) to analyze the epidemiological trends of HP infection prevalence in a symptomatic population in this region of Romania by comparing with previous published data.

\section{Patients and methods}

Study design and setting. A retrospective study was performed including 414 patients who attended a secondary center, 'Salvosan Ciobanca' Medical Center from Zalău, Salaj, Romania between 2014 and 2018.

Participants. Patients with dyspeptic symptoms presented either by their own initiative or were referred by their general practitioners for evaluation of the presence of IgG anti-HP antibodies, were included in the study. There was no exclusion criteria.

Variables. The main outcome measure was the serology test result for HP antibodies. As predictors, we collected data on age, sex, place of residence, and the year of serology test.

Serology analysis. Serology testing was made using immunochromatographic Laboquiq and Intermedical test kit for IgG anti-HP antibodies, and a result of $>20$ was considered positive. All tests were processed according to the manufacturer's recommendations. The data were collected from the hospital archives.

Statistical analysis. Qualitative data are presented by counts and percentages, and normally distributed continuous data as means and standard deviation. Associations between qualitative variables were checked with Chi-square test. Comparisons between two groups regarding normally

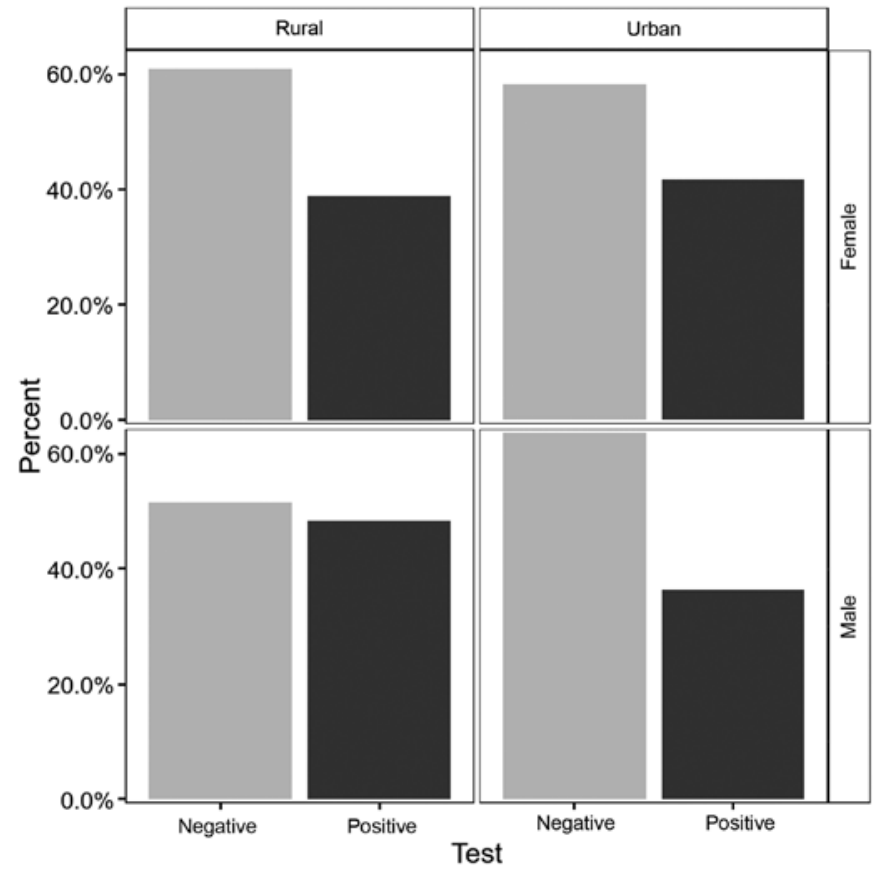

Figure 1. HP test distribution results according to sex and place of residence.

distributed continuous data were performed with independent samples t-test. Tests were presented as two-tailed P-value, of 0.05 level of confidence. The statistical analysis was made using the program R Environment for statistical computing and graphics (R Foundation for Statistical Computing, Vienna, Austria) version 3.2.1.

\section{Results}

Demographic characteristics of the study population. Of the 414 patients, $42.2 \%$ (175 patients) were from rural areas and $57.7 \%$ (239 patients) from urban places. Regarding sex distribution $63.8 \%$ were females (264 persons) and $36.2 \%$ males (150 persons). The mean age of the group was 45.89 , ranging from 6 years to 97 years. The patients characteristics and the prevalence of infection are shown in Table I.

Prevalence of HP infection. Of the 414 patients, $40.8 \%(n=169)$ had positive IgG anti-HP and 59.2\% $(\mathrm{n}=245)$ had negative $\mathrm{IgG}$ anti-HP.

HP prevalence by sex, age group and place of residence. In terms of the positivity of the tests, the percentages were about the same with a slightly higher values among males $41.33 \%$ versus females $40.53 \%$. The differences were not statistically significant $(\mathrm{P}=0.87)$. There was a higher prevalence of the positivity in the rural area $(42.29 \%)$ versus $(39.75 \%)$ in the urban area, but with no statistically significant differences $(\mathrm{P}=0.6)$. The prevalence of infection according to sex and place of residence is displayed in Fig. 1.

The group with negative tests had younger mean age with 2.26 (95\% CI, 1.01-5.53) years, than the group with positive tests, a difference that did not reach the level of significance $(\mathrm{P}=0.176)$. Fig. 2 shows the test result distribution according to age and sex. 
Table I. Prevalence of infection and patients characteristics.

\begin{tabular}{lccc}
\hline Test & All $(\mathrm{n}=414)$ & Positive $(\mathrm{n}=169)$ & Negative $(\mathrm{n}=245)$ \\
\hline Age (years), mean (SD) & $45.89(17.24)$ & $47.22(15.27)$ & $44.97(18.45)$ \\
Age groups (years), $\mathrm{n}(\%)$ & $16(3.86)$ & $3(1.78)$ & 0.176 \\
$<18$ & $59(14.25)$ & $18(10.65)$ & $(5.31)$ \\
$18-29$ & $85(20.53)$ & $35(20.71)$ & $41(16.73)$ \\
$30-39$ & $92(22.22)$ & $41(24.26)$ & $50(20.41)$ \\
$40-49$ & $71(17.15)$ & $40(23.67)$ & $31(20.82)$ \\
$50-59$ & $47(11.35)$ & $14(8.28)$ & $33(13.47)$ \\
$60-69$ & $44(10.63)$ & $18(10.65)$ & $26(10.61)$ \\
$\geq 70$ & $18-29: 59 / 414(14.25)$ & $107(63.31)$ & $157(64.08)$ \\
Sex, $\mathrm{n}(\%)$ & $264(63.77)$ & $62(36.69)$ & $88(35.92)$ \\
Female & $150(36.23)$ & & 0.873 \\
Male & & $95(56.21)$ & $144(58.78)$ \\
Place of residence, $\mathrm{n}(\%)$ & $239(57.73)$ & $74(43.79)$ & $101(41.22)$ \\
Urban & $175(42.27)$ & & 0.604 \\
Rural & & & \\
\hline
\end{tabular}

$\mathrm{SD}$, standard deviation.

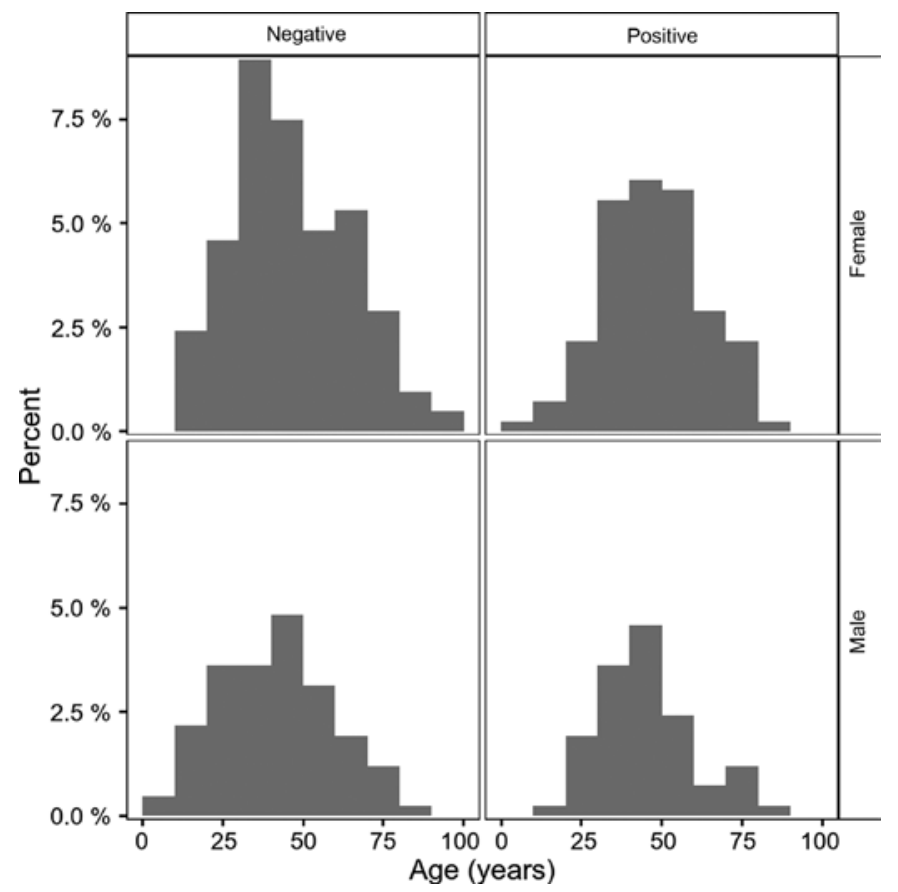

Figure 2. Distribution of the HP test results according to age and sex.

\section{Discussion}

The present study shows that $>40 \%$ of dyspeptic patients had or have HP infection. Comparing these values with those of previous studies on the prevalence of HP infection from the same region, it seems that they are declining. In medical literature there is no clear country wide recent data on this topic.

There are some interesting studies regarding the pediatric population and HP infection from Romania. In 2002, an epidemiological study was performed on dyspeptic children from northwestern region of Romania. The authors included 267 subjects, aged between 5-18 years, and the presence of infection was considered if both urease test and histological staining revealed the infection. The results showed a high prevalence of infection of $40 \%$ (16). In our study, from 16 patients $<18$ years, 3 of them $(1.7 \%)$ had a positive HP infection test.

One year later, in 2003, another study by Miller et al was published regarding the seroprevalence of antibodies to HP in Romanian adopted children, with age between 4 months and 16 years. A high prevalence was found for Romanian children of $20 \%$ (17).

A similar study published in 2018 also reported a high prevalence of HP infection among pediatric population (almost 25\%) from 7,100 children studied from Cluj-Napoca, Romania (18).

An epidemiological study on asymptomatic subjects, from the western part of the county, Timisoara, using a serological test, found high rates of infection in the adult population in 2003. The study population consisted of 960 employees, and it was standardized for age and sex distribution of the western region population. The prevalence of HP infection in the adult population was $68.5 \%$. The prevalence by age group were: $18-30$ years, $65.3 \%$; in group $31-40$ years, $71.6 \%$; in group 41-50 years, 75\%; and in group 51-60 years, $88.7 \%$ (19). Even though the study was done on a smaller population (416 vs. 960), comparing the data, to our study the prevalence of HP infection was lower, as follows: in the group age 18-30 years, $10.6 \%$; in group 30-40 years, $20.7 \%$; in group 40-50 years, $24.2 \%$; and in group 50-60 years, $23.6 \%$.

In 2008 a study targeting the prevalence of HP infection was done in Cluj-Napoca including 955 patients. The global prevalence was $29.7 \%$. The highest prevalence was seen in the age group $>80$ years $(42.8 \%)$, followed by the group 21-30 years $(38.8 \%)$ and the group $41-50$ years (35.9\%). Also, 
Table II. Epidemiological trends of HP prevalence among symptomatic patients in Nortwestern Romania.

\begin{tabular}{lcccc}
\hline Population age (years) & Prevalence $(\%)$ & Reported year & Refs. & Methodology \\
\hline$<20$ & 50 & 1994 & $(21)$ & Urease test \\
$21-30$ & 78.1 & 1994 & $(21)$ & Urease test \\
$31-40$ & 85.9 & 1994 & $(21)$ & Urease test \\
$41-50$ & 80.3 & 1994 & $(21)$ & Urease test \\
$71-80$ & 61.5 & 1994 & $(21)$ & Urease test \\
$5-18$ & 40 & 2003 & $(19)$ & Urease test and histology \\
$18-30$ & 65.3 & 2003 & $(19)$ & Urease test and histology \\
$31-40$ & 71.6 & 2003 & $(19)$ & Urease test and histology \\
$41-50$ & 75 & 2003 & $(19)$ & Urease test and histology \\
$21-30$ & 51.71 & 2009 & $(22)$ & C14-Urease breath test \\
$<20$ & 63.7 & 2017 & $(24)$ & Urease test and IgG anti-HP \\
$20-29$ & 66.22 & 2017 & $(24)$ & Urease test and IgG anti-HP \\
$50-69$ & 61.78 & 2017 & $(24)$ & Urease test and IgG anti-HP \\
$21-30$ & 38.8 & 2018 & $(20)$ & Urease test \\
$41-50$ & 35.9 & 2018 & $(20)$ & Urease test \\
$>80$ & 42.9 & 2018 & $(20)$ & Urease test \\
$<18$ & 1.78 & 2019 & Our study & IgG-anti-HP \\
$18-30$ & 10.65 & 2019 & Our study & IgG-anti-HP \\
$31-40$ & 20.7 & 2019 & Our study & IgG-anti-HP \\
$41-50$ & 24.26 & 2019 & Our study & IgG-anti-HP \\
$>70$ & 10.65 & Our study & IgG-anti-HP \\
\hline
\end{tabular}

the prevalence of HP infection was significantly higher in rural areas (20).

In a study published in 2014 by Ciobanu et al (23), a comparison regarding the data on HP epidemics from Romania was made to see if the tendency of the prevalence was decreasing. The first comparison was made in the same category of age, in different periods of time. The second comparison followed the prevalence trends over 15 years in the same population. In the group of children, a $10 \%$ decreased prevalence was noted from 1994 to 2003. Also, in young adults (20-30 years) from 1994 until 2009, the prevalence dropped significantly from $78.1 \%$ (21) to $51.7 \%$ (22). A similar prevalence was observed in the youngest group, followed over time: $50 \%$ in 1994, without a significant increase in 2009: $51.71 \%$. For this age group, the rate of infection in adult life is very small. These data may reflect the first epidemiological trends of decreasing prevalence of infection in Romania.

Our data show a small difference between patients with HP infection from urban areas compared with rural areas. In urban areas people are more likely to have a higher education and easier access to medical care.

In a study from 2017 made in Craiova on 1,525 dyspeptic patients, in which the prevalence of HP infection was $63.67 \%$, there were no significant percentage differences of the HP infection between the patients from urban area and those from rural area (24). In the study the prevalence of HP infection increased with age, the percentages by age groups were: age $<20$ years, $2.16 \%$; group $20-29$ years, $12.36 \%$; group 30-39 years, $16.7 \%$; group $40-49$ years, $17.1 \%$; group $50-59$ years, $20.7 \%$; and in group $60-60$ years, $17.9 \%$. Comparing the data with those of the present study, the global prevalence (63.67 vs. $40.8 \%)$ and the prevalence in all age groups were lower. The epidemiological trends of HP prevalence in Romania is presented in Table II.

We argue that this decrease in the prevalence of HP infection over time could be attributed to better socio-economic conditions. The fact that patients have easier access to medical services, that more investigation are made when they show various dyspeptic symptoms and due to the existence of non-invasive methods of diagnosing this infection (serology, stool samples, respiratory test), with a more rapid treatment could explain the decreasing trends.

This decreasing prevalence of HP infection is correlated with the evolution of gastric cancer. An epidemiological study published in 2018, regarding the gastric cancer mortality in Romania, in period 1955-2012 shows a decreasing trend in both sexes; from $17.8 \%$ in 1990 to $15.2 \%$ in 2008 and $13 \%$ in 2012. Regarding gastric cancer incidence in period 2008-2012 it seems to be increased in males from $15.9 \%$ in 2008 to $16.3 \%$ in 2012 and appears to be stabilized in females; $5.8 \%$ in 2008 with the same value in 2012 (25).

Our study has several limitations. First, we would have preferred a larger sample, but routine HP screening is not usual in this area. Secondly, as data were collected from HP testing registry, we were not able to record detailed symptoms, the history of HP infection, the followed treatment, and the endoscopic findings. Therefore, further studies should be undertaken. A more detailed analysis on epidemiological trends in Romania in this region, over the last 30 years will be of great interest. However, the present results bring novel 
information on HP infection prevalence in this area, which was the main objective of our study.

This study shows that, among dyspeptic patients from Northwestern part of Romania, $>40 \%$ have or had a positive HP infection. Comparing our results with those of previous studies on the prevalence of HP infection from the same region, we were able to signal a decline in prevalence in $\mathrm{HP}$ infection over a 30-year interval.

\section{Acknowledgements}

Not applicable.

\section{Funding}

No funding was received.

\section{Availability of data and materials}

The datasets used and/or analyzed during the current study are available from the corresponding author on reasonable request.

\section{Authors' contributions}

ALC designed the study, acquired and analyzed the data, and drafted the manuscript. DLD conceived the study and contributed to the analysis of the data and the writing of the manuscript. PC participated in the acquisition and analysis of the data, and reviewed the manuscript. DCL performed the statistical analysis and reviewed the manuscript. All authors had major intellectual contribution to deserve authorship. All authors read and approved the final manuscript.

\section{Ethics approval and consent to participate}

Not applicable.

\section{Patient consent for publication}

Not applicable.

\section{Competing interests}

The authors declare that they have no competing interests.

\section{References}

1. Malfertheiner P, Megraud F, O'Morain CA, Gisbert JP, Kuipers EJ, Axon AT, Bazzoli F, Gasbarrini A, Atherton J, Graham DY, et al; European Helicobacter and Microbiota Study Group and Consensus panel: Management of Helicobacter pylori infection the Maastricht V/Florence Consensus Report. Gut 66: 6-30, 2017.

2. Cheng H, Hu F, Zhang L, Yang G, Ma J, Hu J, Wang W, Gao W and Dong X: Prevalence of Helicobacter pylori infection and identification of risk factors in rural and urban Beijing, China. Helicobacter 14: 128-133, 2009.

3. Klein PD, Graham DY, Gaillour A, Opekun AR and Smith EO; Gastrointestinal Physiology Working Group: Water source as risk factor for Helicobacter pylori infection in Peruvian children. Lancet 337: 1503-1506, 1991.

4. Hopkins RJ, Vial PA, Ferreccio C, Ovalle J, Prado P, Sotomayor V, Russell RG, Wasserman SS and Morris JG Jr: Seroprevalence of Helicobacter pylori in Chile: Vegetables may serve as one route of transmission. J Infect Dis 168: 222-226, 1993.
5. Goodman KJ, Correa P, Tenganá Aux HJ, Ramírez H, DeLany JP, Guerrero Pepinosa O, López Quiñones M and Collazos Parra T: Helicobacter pylori infection in the Colombian Andes: A population-based study of transmission pathways. Am J Epidemiol 144: 290-299, 1996.

6. Brown LM: Helicobacter pylori: Epidemiology and routes of transmission. Epidemiol Rev 22: 283-297, 2000.

7. Hu Y, Wan JH, Li XY, Zhu Y, Graham DY and Lu NH: Systematic review with meta-analysis: The global recurrence rate of Helicobacter pylori. Aliment Pharmacol Ther 46: 773-779, 2017.

8. Venneman K, Huybrechts I, Gunter MJ, Vandendaele L, Herrero R and Van Herck K: The epidemiology of Helicobacter pylori infection in Europe and the impact of lifestyle on its natural evolution toward stomach cancer after infection: A systematic review. Helicobacter 23: e12483, 2018.

9. Hooi JKY, Lai WY, Ng WK, Suen MMY, Underwood FE, Tanyingoh D, Malfertheiner P, Graham DY, Wong VWS, Wu JC, et al: Global prevalence of Helicobacter pylori infection: Systematic review and meta-analysis. Gastroenterology 153: 420-429, 2017.

10. Roberts SE, Morrison-Rees S, Samuel DG, Thorne K, Akbari A and Williams JG: The prevalence of Helicobacter pylori and the incidence of gastric cancer across Europe (Review). Aliment Pharmacol Ther 43: 334-345, 2016.

11. Andreica V, Dumitraşcu D, Sască N, Toganel E, Suciu A, Drăghici A, Pascu O, Sască C, Suciu M, Andreica M, et al: Helicobacter-like organisms in gastroduodenal diseases. Gastroenterol Clin Biol 14: 437-441, 1990.

12. Chen TS, Li FY, Chang FY and Lee SD: Immunoglobulin G antibody against Helicobacter pylori: Clinical implications of levels found in serum. Clin Diagn Lab Immunol 9: 1044-1048, 2002.

13. Lahner E, Bordi C, Di Giulio E, Caruana P, D'Ambra G, Milione M, Grossi C, Delle Fave G and Annibale B: Role of Helicobacter pylori serology in atrophic body gastritis after eradication treatment. Aliment Pharmacol Ther 16: 507-514, 2002.

14. Pérez-Pérez GI, Cutler AF and Blaser MJ: Value of serology as a noninvasive method for evaluating the efficacy of treatment of Helicobacter pylori infection. Clin Infect Dis 25: 1038-1043, 1997.

15. Cutler A, Schubert A and Schubert T: Role of Helicobacter pylori serology in evaluating treatment success. Dig Dis Sci 38: 2262-2266, 1993.

16. Serban R, Grigorescu-Sido P, Gheban D and Kiss E: Helicobacter pylori gastritis in children: Endoscopical and histological aspects. Rom J Gastroenterol 11: 297-301, 2002.

17. Miller LC, Kelly N, Tannemaat M and Grand RJ: Serologic prevalence of antibodies to Helicobacter pylori in internationally adopted children. Helicobacter 8: 173-178, 2003.

18. Domşa T, Gheban D, Rădulescu A and Borzan C: Preliminary research on Helicobacter pylori infection in hospitalized children from northwestern Romania. J Mol Biol 1: 119-120, 2018.

19. Sporea I, Popescu A, van Blankenstein M, Sirli R, Focşea M and Dănilă M: The prevalence of Helicobacter pylori infection in western Romania. Rom J Gastroenterol 12: 15-18, 2003.

20. Prundus C, Ciobanu L, Bolboacă S, Tantău M, Matei D, Cruciat C, Pojoga C and Andreica V: The evolution over time of the prevalence of Helicobacter pylori infection among patients with dyspeptic syndrome - the experience of a tertiary center in Romania. Med Connect 13: 23-27, 2018.

21. Andreica V and Andreica M: Helicobacter pylori infection in stomach and duodenal diseases. Hipocrate, Sibiu, pp10-25, 1994.

22. Ciobanu L, Prundus R, Diaconu B: Epidemiological trends of Helicobacter pylori prevalence in central-western part of Romania. J Gastrointest Liv Dis 18: 17, 2009.

23. Ciobanu L, Taulescu M and Dumitrascu DL: Helicobacter pylori in Romania: Epidemiology, diagnosis and treatment. In: Helicobacter pylori: A Worldwide Perspective, 2014. Buzas GM (ed). Vol 1. Bentham Science Publishers, Oak Park, IL, pp183-201, 2014.

24. Olar L, Mitrut P, Florou C, Mălăescu GD, Predescu OI, Rogozea LM, Mogoantă L, Ionovici N and Pirici I: Evaluation of Helicobacter pylori infection in patients with eso-gastro-duodenal pathology. Rom J Morphol Embryol 58: 809-815, 2017.

25. Valean S, Chira R and Dumitrascu D: Epidemiological trends in digestive cancers in Romania, 1955-2012, compared to alcohol consumption. Correlation or coincidence? Clujul Med 91: 376-386, 2018

This work is licensed under a Creative Common Attribution-NonCommercial-NoDerivatives 4.0 International (CC BY-NC-ND 4.0) License. 